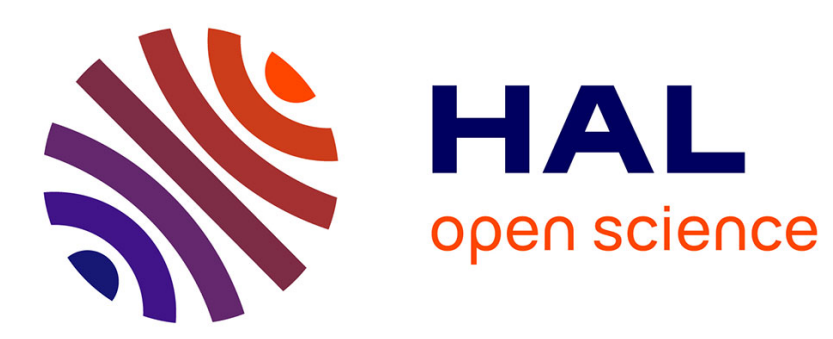

\title{
Epicure, dieu et image de dieu : une autarcie extatique
}

Renée Koch Piettre

\section{To cite this version:}

Renée Koch Piettre. Epicure, dieu et image de dieu : une autarcie extatique. Revue de l'histoire des religions, 1999, 216 (1), pp.5-30. 10.3406/rhr.1999.1110 . halshs-02433806

\section{HAL Id: halshs-02433806 \\ https://shs.hal.science/halshs-02433806}

Submitted on 9 Jan 2020

HAL is a multi-disciplinary open access archive for the deposit and dissemination of scientific research documents, whether they are published or not. The documents may come from teaching and research institutions in France or abroad, or from public or private research centers.
L'archive ouverte pluridisciplinaire HAL, est destinée au dépôt et à la diffusion de documents scientifiques de niveau recherche, publiés ou non, émanant des établissements d'enseignement et de recherche français ou étrangers, des laboratoires publics ou privés. 


\section{Epicure, dieu et image de dieu : une autarcie extatique} Renée Piettre

\section{Citer ce document / Cite this document :}

Piettre Renée. Epicure, dieu et image de dieu : une autarcie extatique. In: Revue de l'histoire des religions, tome $216, \mathrm{n}^{\circ} 1$, 1999. pp. 5-30;

doi : https://doi.org/10.3406/rhr.1999.1110

https://www.persee.fr/doc/rhr_0035-1423_1999_num_216_1_1110

Fichier pdf généré le 04/01/2019 


\section{Résumé}

L'usage abondamment attesté chez les Épicuriens de portraits de leurs fondateurs toujours très ressemblants entre eux s'explique par l'amitié idéalement gémellaire entre Métrodore et Epicure, premier maillon de la chaîne des amis du Jardin, attaché lui-même à un modèle divin qui est le principe et la fin du bonheur épicurien. Or le zèle à imiter les dieux, qui fait de l'épicurisme une véritable réforme religieuse, suppose chez les sectateurs les plus avancés une mutation intérieure et même somatique, dont le mécanisme s'analyse tant dans la théorie de la physique des dieux que dans l'affectivité débordante d'Épicure : il consiste en un flux continu d'images mentales ou visuelles qui s'échangent dans l'effusion de l'amitié. L'être des dieux trouve dans ce commerce fusionnel un aliment ininterrompu et le sage y puise, dans l'extase même, son indestructible sérénité.

\section{Abstract \\ Epicurus : god and image of god. Ecstatic "autarkeia".}

Among the Epicureans the most testified use of their founders' quite similar portraits can be explained by an ideal twin friendship between Epicurus and Metrodorus, which is the first link in the chain of the Kepos' friends, connected to the divine model, principle and aim of the epicurean happiness. Now the zeal for gods imitation, through which epicurism has become a real religious reformation, presupposes that the most advanced disciples internally and even physically move. The mecanism of this transformation can be analysed both in the theory of gods' physic and in Epicurus' overflowing affectivity. It consists in a continuous flood of mental or visual "eidola" which men or (and) gods send to each other in a burst of friendship. The being of the gods finds in this unifying exchange a neverending nutriment, while the sage draws from it right in ecstasy his standing serenity. 


\section{RENÉE PIETTRE}

École pratique des Hautes Études,

Sciences religieuses, Paris

\section{Épicure, dieu et image de dieu : une autarcie extatique}

L'usage abondamment attesté chez les Épicuriens de portraits de leurs fondateurs toujours très ressemblants entre eux s'explique par l'amitié idéalement gémellaire entre Métrodore et Épicure, premier maillon de la chaîne des amis du Jardin, attaché lui-même à un modèle divin qui est le principe et la fin du bonheur épicurien. Or le zèle à imiter les dieux, qui fait de l'épicurisme une véritable réforme religieuse, suppose chez les sectateurs les plus avancés une mutation intérieure et même somatique, dont le mécanisme s'analyse tant dans la théorie de la physique des dieux que dans l'affectivité débordante d'Épicure: il consiste en un flux continu d'images mentales ou visuelles qui s'échangent dans l'effusion de l'amitié. L'être des dieux trouve dans ce commerce fusionnel un aliment ininterrompu et le sage y puise, dans l'extase même, son indestructible sérénité.

\section{Epicurus : god and image of god. Ecstatic autarkeia.}

Among the Epicureans the most testified use of their founders' quite similar portraits can be explained by an ideal twin friendship between Epicurus and Metrodorus, which is the first link in the chain of the Kepos' friends, connected to the divine model, principle and aim of the epicurean happiness. Now the zeal for gods imitation, through which epicurism has become a real religious reformation, presupposes that the most advanced disciples internally and even physically move. The mecanism of this transformation can be analysed both in the theory of gods' physic and in Epicurus' overflowing affectivity. It consists in a continuous flood of mental or visual eidola which men or (and) gods send to each other in a burst of friendship. The being of the gods finds in this unifying exchange a neverending nutriment, while the sage draws from it right in ecstasy his standing serenity.

Revue de l'histoire des religions, 216-1/1999, p. 5 à 30 
Nous avons étudié, dans un article que nous voudrions étroitement complémentaire de celui-ci ${ }^{1}$, un passage du Contre Colotès de Plutarque $(1117 b-f)$ : on y voit, d'après un fragment d'une lettre d'Épicure citée par l'auteur, Colotès, qui fut un disciple d'Épicure dès les premières années de l'école à Lampsaque, se jeter aux genoux de son maître en un geste de vénération qui suscita immédiatement, et comme en miroir, un acte d'adoration identique de la part d'Épicure. Nous avons interprété cette scène comme l'illustration d'une phase essentielle de l'initiation épicurienne : la reconnaissance de la divinité du maître et l'accès simultané $\mathrm{du}$ disciple à cette même divinité. Cette interprétation s'appuyait notamment sur de nombreux autres témoignages de l'exaltation affective cultivée dans le Jardin. Mais elle requiert un réexamen de la doctrine de l'amitié, entendue comme un processus de réciprocité dans l'échange d'images bienheureuses, s'articulant d'une part avec l'usage abondamment attesté des portraits des philosophes dans la secte, d'autre part avec la théorie de la perception et de la physique des dieux selon Épicure. C'est ce réexamen que nous voudrions aborder ici, d'une manière plus programmatique qu'exhaustive. Nous espérons souligner par là le sérieux renouvelé avec lequel il conviendrait de considérer de nombreuses approches critiques tendant à mettre en lumière le caractère foncièrement religieux de la vie quotidienne et des enseignements du Jardin.

Images des sages images des dieux

L'Épicurien de l'Antiquité adorait des images. Celles des dieux, qui se présentent d'elles-mêmes, comme en épiphanie, à l'esprit des sages capables d'accéder à leur contem-

1. Lalies, 18, 1998, p. 185-202. 
plation; mais aussi les statues des sages fondateurs euxmêmes ${ }^{2}$.

Les portraits des philosophes étaient largement diffusés à travers le monde gréco-romain. Rien de plus répandu cependant que les portraits d'Épicure et des maîtres qui

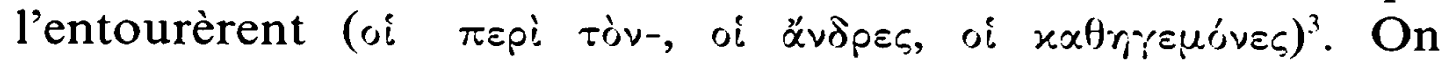
connait $^{4} 21$ bustes de l'obscur Hermarque, le successeur immédiat d'Épicure, contre 18 seulement d'Aristote. Les plus riches se les offraient en marbre, les autres n'avaient que des images peintes, ou portaient l'image gravée d'Épicure sur une bague, ou la retrouvaient au fond de leur coupe à boires. Bernard Frischer a consacré à ces images un ouvrage impor$\operatorname{tant}^{6}$, qui discerne chez les Épicuriens un véritable fétichisme (mot que l'auteur utilise en un sens très soigneusement défini).

Tous les portraits d'Épicure étaient faits sans doute à la ressemblance d'une image mère ${ }^{7}$, qui peut avoir été la statue assise (trônante) d'Épicure que la secte avait fait ériger en public à Athènes entre 280 et 250 av. J.-C. ${ }^{8}$, ou encore celle que F. Poulsen estimait avoir originellement orné le Jardin".

Mais cette dernière statue n'était pas seule. Elle faisait groupe avec les images des deux autres fondateurs, Métrodore

2. Cicéron, De finibus V, 1, 3 ; Pline l'Ancien, Histoire naturelle 35, 5 ; Usener, Epicurea 390 (= un passage d'Origène, Contre Celse, VII, 66, p. 386, Koesch., où l'auteur paraît penser qu'on adressait des prières à des images d'Épicure).

3. Cf. Francesca Longo Auricchio, La scuola di Epicuro, Cronache Ercolanesi 8, 1978, p. 21-37. Il s'agit du quatuor des fondateurs Épicure, Métrodore, Hermarque, Polyène. Colotès (cf. Bernard Frischer, The Sculpted Word. Epicureanism and Philosophical Recruitment in Ancient Greece, University of California Press, Berkeley, Los Angeles, London, 1982, p. 88$89)$ avait aussi ses portraits.

4. Selon Bernard Frischer, 1982, p. 91.

5. Cicéron, De Finibus V, 1, 3 ; Pline l'Ancien, Histoire naturelle 35, 5.

6. Cf. n. 3, supra.

7. Cf. Henning Wrede, Bildnisse epikureischer Philosophen, Mitteilungen des Deutschen Archäologischen Instituts (Athenische Abteilung), 1982, p. 235-245, ici p. 237.

8. B. Frischer, 1982, "Préface ", p. XVI sq.

9. F. Poulsen, Ikonographische Miscellen, Copenhague, 1931, p. 77 sq. 
et Hermarque, selon V. Kruse-Berdold ${ }^{10}$ : Épicure était assis sur un trône à pieds de lions, Métrodore sur un klismos garni de coussins, Hermarque sur un banc grossier. Elle faisait couple, estimait quelques décennies plus tôt $F$. Poulsen, avec une statue symétrique représentant le plus cher ami d'Épicure, celui qui le seconda de plus près dans son activité évangélique, Métrodore de Lampsaque: les deux statues étaient quasi semblables, à ceci près qu'Épicure était montré juste après la lecture d'un rouleau tenu dans sa main gauche, et Métrodore sur le point de lire un rouleau tenu dans sa main droite. Il est d'ailleurs souvent difficile, constatent les spécialistes $^{11}$, de distinguer un portrait d'Épicure d'un portrait de Métrodore, ou d'Hermarque, successeur d'Épicure (Métrodore étant mort avant le fondateur).

Nous ne discuterons pas ici un problème archéologique : il nous suffit que les auteurs soient d'accord pour reconnaître ces étonnantes ressemblances du portrait de l'un au portrait de l'autre.

Le risque de confusion autant que les subtiles différences s'expliquent sans difficulté, si l'on veut bien entrer dans l'esprit de l'ascèse épicurienne: selon le témoignage de Sénèque rapportant un mot du maître, Épicure a su de luimême trouver le chemin de l'ataraxie et se conformer au modèle divin; Métrodore en disciple doué a su suivre ses traces ; Hermarque, plein de bonne volonté sans doute, a été hissé et tiré sur la même voie ${ }^{12}$, et c'est ainsi que tous trois sont parvenus au même but, l'imitation du divin, et que la ressemblance avec le même modèle les rend tous trois semblables entre eux, même s'il faut admettre chaque fois ce léger décalage ${ }^{13}$.

10. Veronika Kruse-Berdold, Kopienkritische Untersuchungen zu den Porträts des Epikur, Metrodor und Hermarch, Diss. Göttingen, 1975, p. 150 sq., 178 sq. et 207 sq.

11. B. Frischer, 1982 , p. $122-123$ et notes.

12. Cf. Sénèque, Lettres à Lucilius 52, 3.

13. Francesca Longo Auricchio (Ermarco. Frammenti, Naples, 1988, p. 27 sq.) réhabilite Hermarque et prétend qu'il n'est en rien tenu pour inférieur à Métrodore, la différence indiquée dans Sénèque étant de tempéra- 


\section{Un hermès double: Épicure Métrodore}

Cette remarquable application de l'effort philosophique vers la semblance au dieu réalise, en fait, le but ultime de la doctrine du Jardin, tel qu'il est énoncé à la fin de la Lettre à Ménécée: "Tu vivras comme un dieu parmi les hommes. " Épicure a donné au modèle divin un visage humain en tant que le divin est en effet selon lui un modèle imitable, et le miracle de cette transfiguration, dans son processus et quasi sa recette, peut alors être lui-même mis en image dans l'imitation du maître par le disciple, mieux, dans une espèce de scissiparité du modèle. Or de cette dernière nous trouvons une première illustration saisissante dans un célèbre hermès bifrons de la Stanza dei filosofi du Musée du Capitole à Rome, représentant d'un côté le visage d'Épicure, et du côté opposé le visage de Métrodore. Bernard Frischer ${ }^{14}$ vante l'extraordinaire magnétisme de cette image qui, malgré d'innombrables transformations dans l'arrangement de la salle, n'a jamais quitté la place centrale où elle focalise tous les regards. Cette image nous rappelle qu'Épicure ne s'est pas offert en modèle comme un solitaire au-dessus de la mêlée, mais comme un être double, un peu, pour employer une comparaison imparfaite, comme ces premiers hommes parfaitement heureux du mythe d'Aristophane dans le Banquet de Platon : comme un couple d'amis, comme une totalité de bonheur, à la fois close et ouverte à la façon de deux maillons d'une chaîne faite pour se prolonger par le moyen de maillons supplémentaires attirés par le magnétisme puissant de cette amitié mère.

ment et non de mérite (au contraire, dit Sénèque 52, 4, Hermarque a plus de mérite que Métrodore). Voir cependant infra. H. Wrede, 1982, estime que d'après les bustes Hermarque ressemble à Épicure plus que Métrodore, et qu'il est par conséquent le disciple le plus fidèle (p. 241 sq.). Cependant à ses yeux le portrait de Métrodore «traduit de la façon la plus transparente les maximes éthiques du Jardin " (p. 242). Hermarque dépend davantage du maître, Métrodore a plus d'autonomie.

14. B. Frischer, 1982, "Préface ", p. XIII. 
Cette statue double, cet hermès bifrons doit en effet nous apparaître comme le symbole du pilier-maître de cette communauté épicurienne qui se perpétua durant sept siècles et accueillit des millions d'adeptes. L'épicurisme est né de cette amitié-là et ne s'est répandu que par la fécondité de ce couple premier, de cet hermaphrodite étrange.

On en trouverait bien d'autres indices. La secte, telle du moins que l'avait établie Épicure, était fondée non sur le maître Épicure mais bien sur le couple formé par Épicure et Métrodore :

$1 /$ On repère de nombreux textes et passages où le nom de Métrodore accompagne celui d'Épicure ${ }^{15}$.

2 / Dans certains passages de ce type, Épicure paraît reverser directement la vénération qu'on lui adresse sur Métrodore ou sur ses proches.

- Ainsi dans Cicéron, De finibus 30, 96, citant une lettre à Hermarque d'Épicure mourant : "Pour toi, en conformité avec les sentiments dont, depuis ta première jeunesse, tu as été animé à l'égard de ma personne et de la philosophie, fais en sorte de veiller sur les enfants de Métrodore. $»^{16}$

- Dans un fragment préservé sur un papyrus d'Herculanum, Épicure fait l'éloge d'un tiers, Leonteus de Lampsaque, un disciple de la première heure, en disant qu'il avait une attitude de grande vénération "aussi bien à mon égard qu'à

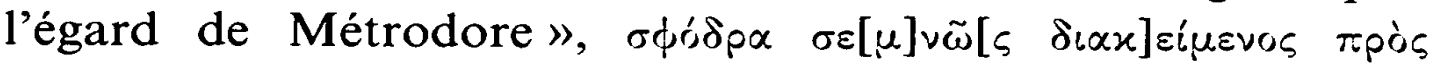

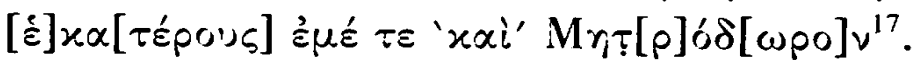

15. Cf. F. Longo Auricchio, 1978, p. 22, n. 8. On peut citer Plutarque, Non posse 1086E, 1087A, 1091E, 1097B, 1103A; Adv. Col. 1108E, 1117B, 1126E, 1127E ; De defectu or. 420D ; Lucien, Alex. 17 ; Athénée, Deipn. VII, 279 sq. ; Strabon XIII, 589; Cicéron, Tusc. II, 3, 8; V 37, 108; De natura deorum I, 31, 86;33, 93 ; 40, 113 ; De finibus I, 7, 25 ; II, 3, 7; 28, 92 ; 30, 98 ; 31, 101 ; Sénèque, Lettres à Lucilius 18,$9 ; 79,16 ; 81,11$. Voir l'opinion de A. Körte, Metrodori Epicurei fragmenta, Jahrbuch für Classische Philologie, Supplement 17, Leipzig, 1890, p. 535.

16. Trad. Jules Martha, Belles Lettres.

17. PHerc. 176, fr. 5, col. XII, cf. Anna Angeli, La scuola epicurea di Lampsaco nel PHERC. 176 (fr. 5, coll. I, IV, VIII-XXIII), Cronache Ercolanesi 18, 1988, p. 27-51, ici p. 34. Mais voir la critique de C. Militello (éd.), Filodemo, Memorie epicuree, Napoli, 1997, p. 49-56. 
- La fête centrale de la communauté, celle qui réunissait les membres de chaque cercle le 20 de chaque mois, si importante que les Épicuriens dans leur ensemble étaient souvent appelés les Eikadistai, les Adeptes de la fête du $20^{18}$, a été fondée, selon la volonté exprimée par Épicure dans son testament, en l'honneur d'Épicure et de Métrodore. Elle n'était pas spécialement destinée à célébrer le souvenir du fondateur, ce qui eût dû se faire à l'anniversaire de sa naissance ${ }^{19}$. Placée en un jour où les Grecs avaient coutume de fêter Apollon $^{20}$, elle ne constitue pas davantage un culte héroïque : elle célèbre un dieu, et ce dieu est expressément désigné comme double, il s'agit d'Épicure et de Métrodore unis par l'amitié ${ }^{21}$. Au mois de Gamélion, la même fête du 20 célébrant l'anniversaire de la naissance du fondateur est cependant, de cette manière, replacée dans la perspective d'une nouvelle naissance, la naissance à l'amitié et à la perfection de la sagesse, comme après une initiation éleusinienne : et justement, c'est un 20 aussi que $\mathrm{s}^{\prime}$ achevaient les Mystères ${ }^{22}$.

18. Eikades : cf. Pierre Boyancé, Sur une épitaphe épicurienne, Revue des Études latines 33, 1955, p. 113-120.

19. Le $20 \mathrm{du}$ mois de Gamélion uniquement : cf. André Laks, Édition critique et commentée de la "Vie d'Épicure" dans Diogène Laërce (X, 1-34), Cahiers de philologie I, Publications de l'Université de Lille III, 1976, p. $1-118$, ici p. 85, n. 21 . Mais cette date est en concurrence avec celle du 10 et celle du 7 du même mois, dans Diogène Laërce 10, 18 et 10, 14 : cf. Diskin Clay, The Cults of Epicurus, Cronache Ercolanesi 16, 1986, p. 11-28; W. Schmid, Epikur, Reallexicon der Antike und Christentum V, 1962, 681819 (= Epicureo e l'epicureismo cristiano, trad. ital. d'Italo Ronca, Antichità classica e cristiana 24, Brescia, 1984), p. 17 de l'édition italienne; et W. Schmidt, Geburtstag im Altertum, Giessen, 1908, p. 43 sq. et 89 . Le 7 est consacré à Apollon, ce dont B. Frischer, 1982 (p. 275 sq.), tire argument pour lire dans le nom d'Épikouros un pseudonyme emprunté à une épiclèse d'Apollon (Épikourios)...

20. H. Steckel, Epikuros, in Pauly-Wissowa, Suppl. 11, 1968, col. 580.

21. Diskin Clay, 1986 , p. 25 , fait remarquer en effet le caractère divin et non héroïque de cette fête : "In striking contrast to the cult of Plato in the Academy, the cult of Epicurus was celebrated both on his birthday, as befitted a heros ktistes, and on the twentieth of each month as befitted a god: deus ille fuit, deus." "

22. Norman W. De Witt, Epicurus and his philosophy, Minneapolis, 1954, p. 50. 
3 / Métrodore est le seul à avoir peut-être collaboré aux écrits dogmatiques fondateurs de la doctrine positive ${ }^{23}$.

4 / Les fidèles réservaient le nom de Père à Épicure, mais aussi à Métrodore et à Hermarque ${ }^{24}$. Aux côtés du maître, Métrodore seul, en qui l'on a pu voir «presque un second Épicure $»^{25}$, fut honoré du nom de sage, sophos, selon Cicéron, De Finibus II, 3, 7 : Épicure, le «seul sage qui, à ma connaissance, ait osé se proclamer sage lui-même. Car pour Métrodore, je ne crois pas qu'il se soit lui-même proclamé sage, mais qu'ainsi nommé par Épicure il ne voulut pas refuser un si beau cadeau ".

Mais on remarque la réticence de Cicéron, qui peut être rapprochée de ce témoignage de Sénèque ${ }^{26}$ : Métrodore, toujours un peu en retrait par rapport à Épicure, avait besoin d'un as entier pour se rassasier, alors qu'Épicure pouvait se contenter de moins d'un as. Les statues trônantes du Jardin selon Poulsen auraient montré ce même décalage - Métrodore commence à lire quand Épicure vient de finir. Il existe, nous l'avons vu, d'Épicure à Hermarque, une hiérarchie qu'Épicure a lui-même explicitée ${ }^{27}$.

23. Notamment par un traité Sur les sensations (Diogène Laërce $X, 24$ ), et par des traités de physique et d'éthique, selon De Witt, 1954, p. 115 (les autres fondateurs et disciples n'ont participé qu'à des écrits de réfutation des théories adverses ou à des ouvrages destinés à célébrer la mémoire d'autres Épicuriens). Philodème, dans le De Pietate reconstitué par Dirk Obbink (Philodemus, On Piety, Part 1, Oxford, 1996), col. 7, citerait coup sur coup, à l'appui de son argumentation, Métrodore et Épicure dans des

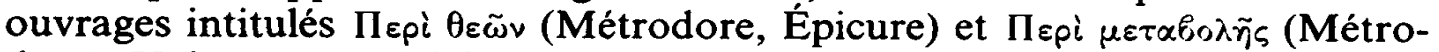
dore). Voir surtout Adele Tepedino Guerra, Il contributo di Metrodoro di Lampsaco alla formazione della theoria del linguaggio, Cronache Ercolanesi 20,1990 , p. 17-26.

24. Selon ce que l'on peut induire d'un passage de la Rhétorique de Philodème (A col. VII. 18-28, vol. I, p. 12, Sudhaus).

25. Cicéron, De finibus 28, 92.

26. Sénèque, Lettres à Lucilius 18, 9.

27. Sénèque, Lettres à Lucilius 52,3 ; cf. supra. 
L'épicurisme, une religion

Le modèle individuel de sagesse voulu et constitué par Épicure, étroitement lié à la communauté qu'il a fait naître par la vertu d'un échange amical fondateur, ne sera cependant apprécié à sa juste mesure que si on l'identifie comme un phénomène religieux.

Cette thèse reste difficile à faire admettre en raison d'un triple préjugé : d'une part toute l'Antiquité a crié à l'impiété, voire à l'athéisme, parce qu'Épicure niait la Providence et, plus largement, toute ingérence des dieux dans les affaires humaines, prétendant en outre supprimer chez ses adeptes la crainte des dieux, promue au rang de monstre à abattre, de mal absolu ; d'autre part, les chrétiens se sont d'autant mieux appliqués à faire d'Épicure leur épouvantail qu'ils lui ont, comme il a été amplement démontré, beaucoup emprunté à leurs débuts ${ }^{28}$; enfin, depuis Gassendi et depuis Marx, nous autres libertins ou matérialistes, qui voyons en Épicure un de nos champions, souffrons difficilement qu'on ait l'air de jeter une ombre sur sa rationalité. Et c'est ainsi que, malgré de

28. Il est d'usage de considérer que Norman W. De Witt (Epicurus and his Philosophy, Minneapolis, 1954, passim) a beaucoup exagéré dans les rapprochements qu'il a prétendu faire entre le christianisme primitif et l'épicurisme, « puisque » l'épicurisme n'est pas une religion, etc. On obtiendrait cependant un résultat assez éloquent en conjuguant les arguments d'Abraham J. Malherbe, Self-definition among Epicureans and Cynics, in B. F. Meyer et E. P. Sanders (eds), Jewish and Christian Self-Definition, vol. 3, Londres, 1982; Hellenistic Moralists and the New Testament, ANRW II, 26 (ed. W. Haase), 235-238 ; R. L. Wilken, Collegia, Philosophical Schools, and Theology, in S. Benko et J. J. O'Rourke (eds), The Catacombs and the Colosseum, 1971, p. 268-291; H. Steckel, 1968 ; R. Jungkuntz, Fathers, Heretics and Epicureans, Journal of Ecclesiastical History 17, 1966, p. 3-10 ; W. Schmid, 1984 ; A. D. Simpson, Epicureans, Christians, Atheists in the Second Century, Transactions and Proceedings of the American Philological Association 72, 1941, p. 372-381. - Notons qu'à la fin du sixième livre des Confessions saint Augustin raconte qu'à l'époque où il cherchait sa voie parmi les arts de vivre qui étaient proposés à ses contemporains il penchait pour l'épicurisme, qui lui paraissait la secte la plus recommandable. 
nombreuses avancées au plus près du but que nous désignons ici, la plupart reculent au dernier moment et parlent qui d'une réforme "morale " (De Witt), qui d'une "quasi-religion" (W. Schmid) ${ }^{29}$, qui d'une opposition radicale entre la rationalité philosophique et la religion supposée irrationnelle ${ }^{30}$.

D'autres pourtant ne reculent pas, ainsi Benjamin Farrington : « It was not a fashionable movement with a merely intellectual appeal. It was a call to a new way of life... Epicureanism was not a politique but a mystique. ${ }^{31}$ Il faudrait citer aussi Paul Rabbow (fort critiqué en son temps) ${ }^{32}$ et Ettore Paratore, A. J. Festugière (l'esprit plutôt que la lettre), Ilsetraut Hadot, Wolfgang Fauth, Pierre Hadot (avec une perspective plus générale sur l'ensemble des sectes philosophiques en Grèce), et, avant eux, Heidel, F. Picavet, C. Pascal ${ }^{33}$. Ber-

29. W. Schmid, 1984, p. 101, tâchant de ne pas basculer dans l'hérésie, est équilibriste jusqu'au prodige : " Pertanto, chi tenti di comprenderla adeguatamente, dovrà da une lato prender sul serio la stilizzazione religiosa dell'Epicureismo (senza cioè cercare di ridurla o magari di eliminarla con frettolose manipolazioni esegetiche), dall'altro lato tener costantemente presente il suo carattere non religioso ma soltanto "quasi-religioso". "

30. Nous renvoyons ici à Claude Lévi-Strauss démontrant l'homogénéité de la pensée mythique et de la pensée rationnelle.

31. Benjamin Farrington, The Faith of Epicurus, London and Edinburgh, 1967, p. 11.

32. Paul Rabbow, Seelenführung. Methodik der Exerzitien in der Antike, München, 1954 (les exercices spirituels des Épicuriens et des Stoïciens préfigurent ceux des chrétiens). Voir les comptes rendus de Leipoldt, ThLZ 80, 1955, p. 540 ; Naumann, ZKTh 77, 1955, p. 247-248 ; Grilli, Paideia 11, 1956, p. 133-135; Luck, Gnomon 28, 1956, p. 268-271; ou dans la Nouvelle Revue de théologie 78, 1956, p. 769.

33. Ettore Paratore, Il fondamento religioso della «metafisica » epicurea, Annali della Scuola Normale di Pisa, 1947, p. 125-148; La problematica sull'epicureismo a Roma, Aufstieg und Niedergang der römischen Welt I.4, 1973, p. 116-204, notamment p. 129-132; A. J. Festugière, Épicure et ses dieux, Paris, $1968^{2}$; Ilsetraut Hadot, Seneca und die griechisch-römische Tradition der Seelenleitung, Berlin, 1969 ; Wolfgang Fauth, Divus Epicurus: Zur Problemgeschichte philosophischer Religiosität bei Lukrez, Aufstieg $u$. Niedergang I.4, 1973, p. 205-225 ; Pierre Hadot, Exercices spirituels et philosophie antique, Paris, $1993^{3}$; Qu'est-ce que la philosophie grecque? (ces deux derniers tendant à tirer vers la sphère religieuse plus que la philosophie épicurienne). Également F. Picavet, De Epicuro novae religionis auctore, Paris, 1889, F. Alcan; W. A. Heidel, Zeitschrift für Religionpsychologie 3, 1910, p. 397 ; C. Pascal, La venerazione degli dèi in Epicuro, Rivista di Filosofia 34, 1906, p. 241-256 et Epicurei e mistici 2, Catania, 1914. 
nard Frischer a introduit dans la discussion des méthodes inédites. De Witt ${ }^{34}$ (quelques corrections qu'il convienne d'apporter $)^{35}$ et $\mathrm{W}$. Schmid restent des auxiliaires très précieux dans la direction qui est la nôtre, cependant que la récente

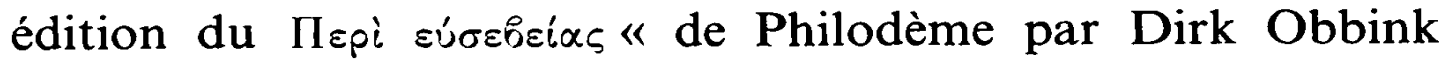
(supra n. 23) apporte sur des sujets tellement obscurs abondance de l'information, clarté et sûreté : ni la piété d'Épicure ni le sérieux de son adhésion à une existence réelle des dieux ne peuvent réellement être mis en doute. Et nous noterons incidemment chez Lemke la définition de la doctrine d'Épicure sur les dieux et sur la religion comme une " théologie de la religion olympienne ${ }^{36}$.

On souligne à l'envi, depuis l'Antiquité, la fidélité de la secte épicurienne à son fondateur ${ }^{37}$. Cela est dû d'abord à la

34. Dont il faut signaler encore Organization and Procedure in Epicurean Groups, Classical Philology 31, 1936, p. 205-211 et Epicurean Contubernium, Transactions and Proceedings of the American Philological Association 67, 1936, p. 55-63.

35. Le déchiffrement toujours en cours des papyri d'Herculanum et de l'inscription de Diogène d'Oenanda ne cesse de préciser et d'étendre notre documentation.

36. D. Lemke, Die Theologie Epikurs, Zetemata 57, Munich, 1973.

37. Voir Sénèque, Ép. 33, 4 ( «Chez ces gens-là, tout ce qu'a dit Hermarque, tout ce qu'a dit Métrodore n'est imputé qu'à un seul... ") ) ; Numenius ap. Eusèbe, Préparation évangélique 114.5, 727D-728A. Il faut naturellement apporter quelques nuances: discussions et désaccords du vivant même d'Épicure, dont la catastrophique défection de Timocrate frère de Métrodore; obscures dissidences, à Rhodes par exemple, sur lesquelles nous sommes progressivement renseignés par le déchiffrement de Philodème, notamment autour de la question de la rhétorique ; évolutions inévitables, liées à l'affinement de la discussion antistoïcienne, au contexte romain, ou bien à la forte personnalité et au talent propre de certains disciples (voir, pour Lucrèce, ne serait-ce que le recours à la versification, et, chez Diogène d'Enanda, l'utilisation d'une forme de publicité de la doctrine fondamentale, par une gigantesque inscription murale offerte aux passants, très différente des écrits à usage interne qui circulaient d'ordinaire). Sur la question voir David Sedley, Philosophical Allegeance in the GrecoRoman World, in Miriam Griffin et Jonathan Barnes (eds), Philosophia togata. Essays on Philosophy and Roman Society, Oxford, 1989, p. 97-119; F. Longo Auricchio et A. Tepedino Guerra, Aspetti e problemi della dissidenza epicurea, Cronache ercolanesi 11, 1981, p. 25-40 ; F. Longo Auricchio, 1978 ; Jean-Marie André, Les écoles philosophiques aux deux premiers siècles de l'Empire, Aufstieg und Niedergang der Römischen Welt II, 36 , p. 5-77. 
présentation même des textes et aux formes de l'enseignement. Toute la doctrine pouvait s'appréhender par paliers, du général au particulier, et se déduire en quelque sorte des propositions fondamentales, de la même façon que la géométrie d'Euclide (un contemporain d'Épicure) découle tout entière des théorèmes fondamentau $x^{38}$. Ramassée dans le tétrapharmakon ou dans les trois lettres conservées par Diogène Laërce, déployée dans les 6 chants de Lucrèce ou détaillée dans les 37 livres de la Physique d'Épicure lui-même, la doctrine ne variait pas. Elle variait d'autant moins qu'Épicure l'avait accompagnée d'une pédagogie, ou plutôt d'une psychagogie elle-même méticuleuse, contraignante et particulièrement efficace ${ }^{39}$ : tant par la répétition - y compris à haute voix - et par la rumination - y compris par des reformulations écrites - que par la discussion et par l'examen de conscience, il s'agissait de parvenir à une adhésion totale et immédiate à des propositions que, simultanément, la raison devait tenir pour évidentes et nécessaires. Cependant cette force extraordinaire de l'épicurisme ne peut s'expliquer que d'une manière : il faut admettre que, de façon très différente des «thiases » philosophiques qui l'ont précédé, par ce dogmatisme de fer si décidé à s'implanter dans les âmes pour leur salut, l'épicurisme est une religion. De la religion il a cette emprise entière sur l'homme entier. Mais une religion qui au lieu de se couler dans le moule existant du thiase religieux, comme l'avaient fait toutes les autres jusque-là ${ }^{40}$, refusait justement ce lien institutionnel trop commode, et vivait sur un

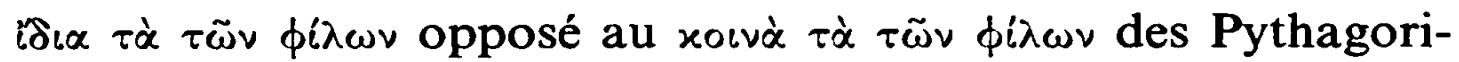

38. Même les contradicteurs connaissent fort bien la doctrine et celle-ci souffre en quelque sorte de l'efficacité pédagogique du maître puisque ses ennemis sont capables de répéter mot pour mot ses propres théorèmes pour les ridiculiser (Cicéron dans le De Natura deorum et le De Finibus ou le Contre Pison, voire dans des lettres à son ami épicurien Atticus ; Plutarque, Origène, Lactance).

39. On se reportera à $P$. Rabbow, 1954 ; N. W. De Witt, 1936 et 1954, I. Hadot, 1969 ; P. Hadot, 1993.

40. Voir Paul-François Foucart, Des Associations religieuses chez les Grecs: Thiases, Éranes, Orgéons, Paris, 1873 ; Pierre Boyancé, Le Culte des Muses chez les philosophes grecs, Paris, 1936. 
ciens $^{41}$. Il faut admettre aussi qu'Épicure est un dieu, et pas seulement en un sens métaphorique, mais par une sorte de révolution organique qui le fait accéder à une forme nouvelle... d'humanité. Nous nous risquons même à penser qu'il pourrait s'être opéré là un véritable phénomène de civilisation. Par la médiation de la divinité d'Épicure naît, en marge de la polis, dans la franchise ${ }^{42}$ la plus grande de chaque sectateur envers lui-même et envers son maître, uniquement appuyé sur les lois de la nature accessibles par le travail de la mémoire et de minutieux examens de conscience ${ }^{43}$, le premier individu autarcique.

$\mathrm{Si}$ les amateurs de philosophie flirtaient généralement avec une école puis l'autre, il n'arrivait quasiment jamais que les adeptes d'Épicure quittent le Jardin. Cela s'explique peutêtre par la clôture de la secte, mais il doit y avoir aussi autre chose, comme une mutation irréversible. On peut être homme et devenir eunuque, mais une fois qu'on est devenu eunuque on ne peut plus revenir en arrière, ironisait une méchante langue.

\section{Comment on devient dieu}

Notre hypothèse, c'est que la mutation dont il s'agit doit

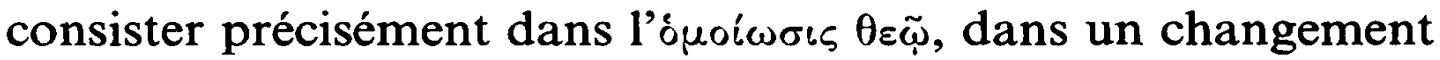
radical d'état mental et, partant, nécessairement physique - selon la théorie matérialiste d'Épicure -, qui s'opère chez le sage lorsqu'il atteint la semblance au dieu. Épicure donne

41. B. Frischer, 1982, p. 42 sq.

42. Cf. le traité de Philodème sur la parrhèsia (P. Herc. 1471:A. Oli-

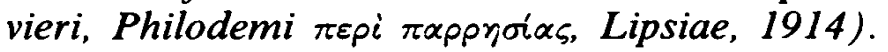

43. Voir, outre les auteurs cités supra, n. 39, F. Zucker, SyneidesisConscientia. Ein Versuch zur Geschichte des sittlichen Bewusstsein im griechischen und im griechisch-römischen Altertum, Jena, 1928; W. Schmid, Contritio und "ultimea linea rerum " in neuen epikureischen Texten, Rheinisches Museum 100,1957, p. 301-327. Le travail sur la conscience est selon W. Schmid, 1984, p. 94, un des domaines où une idée épicurienne est devenue patrimoine commun de l'hellénisme tardif (et, ajouterons-nous, de la civilisation occidentale en général). 
accès aux dieux, en tant qu'il a eu lui-même accès à leur contemplation mentale et qu'il a parachevé son propre bonheur par l'amitié partagée avec Métrodore. Ce point constitue la clef de voûte où la doctrine trouve sa cohésion et son équilibre. En effet, les dieux vivent dans une bienheureuse autarcie, comme les lois de la physique, assise et marchepied de l'éthique, nous le démontrent les premières, et c'est la contemplation de leur exemple, mentalement accessible au sage à volonté (pacata posse omnia mente tueri) ${ }^{44}$, qui lui permet d'atteindre à ce souverain bien qu'est l'ataraxie par laquelle il se découvre l'égal des dieux. Une réciprocité s'établit alors : < Le sage> "nomme les sages amis des dieux et les dieux amis des sages", écrit Philodème (Des dieux III, col. 1, 17-18 Diels).

Mais comment cette doctrine se traduit-elle dans la vie et dans l'organisation du Jardin?

Dans le principe, l'aimantation de l'exemple du sage, luimême aimanté par l'exemple des dieux, est la force qui cimente la communauté épicurienne. "Le sage et les dieux épicuriens sont des moteurs immobiles, comme le dieu d'Aristote, remarquait B. Frischer : ils attirent les autres, en leur transmettant leurs images. $»^{45}$ Mais cette aimantation elle-même emprunte une voie originale, qui est l'amitié: "L'amitié mène sa ronde autour du monde, comme un héraut nous appelant à nous réveiller pour nous estimer bienheureux ", dit la Sentence 52. Des dieux aux sages, une chaîne de l'amitié se forme, une ronde d'amis qui se maintiennent les uns les autres dans leur béatitude par la réciprocité des services amicaux, une réciprocité dont la pérennité est garantie par l'élan en quelque sorte extatique de l'amitié prolongé, au-delà de la mort individuelle, par le souvenir du sage diligemment entretenu au moyen de la diffusion de ses images ou des réunions rituelles et festives de la secte à dates fixes, occasions d'une relecture des textes fondateurs.

44. Lucrèce V, 1203.

45. B. Frischer, 1982, p. 83, cité par Pierre Hadot, Qu'est-ce que la philosophie antique?, p. 345. 
Or nous mettrons l'accent sur le fait que recevoir, par la contemplation, des images (simulacres) du sage ou des dieux, cela constitue pour Épicure (en vertu de sa doctrine intégralement matérialiste) un phénomène physique conduisant à nourrir notre être corporel, à lui conférer l'accroissement et enfin la plénitude de la stabilité dans l'ataraxie. Le Jardin est ainsi le lieu d'une économie communautaire ${ }^{46}$, et nous entendons cette formule ainsi : l'économie épicurienne c'est la régulation des échanges privés, propres (oix<ĩos) de chacun en tant qu'il a besoin de ce qui lui convient en particulier; si elle est communautaire une contradiction apparaît, qui ne peut se résoudre que par la multiplication des échanges privés d'une part, la réduction de l'autre au même d'autre part. C'est ainsi que Lucrèce adresse son immense poème à un seul, en vue de sa conversion. Mais Memmius, une fois converti et devenu l'égal d'Épicure, pourra, en principe, transmettre à son tour à un ami la précieuse doctrine.

C'est avec la même façon de transmettre dans la pratique l'élément vital que les adeptes apportaient à Épicure et aux autres "amis" une contribution financière ou matérielle que par amitié le récepteur redistribuait à son tour aux autres membres, dans les repas en commun et l'entretien ordinaire du jardin comme dans les célébrations des fêtes ${ }^{47}$.

Ici nous avons l'air de prendre pour argent comptant ce qui n'est peut-être qu'analogie verbale. Peut-on vraiment réduire chez Épicure l'éthique à la physique ? Ce point a toujours retenu les commentateurs. "Comme un dieu parmi les hommes " : faut-il s'arrêter à cette comparaison? Mais Épicure n'engage Ménécée à cette vie de quasi-félicité divine que

46. Voir Christian Jensen, Philodemi repi oixovouias qui dicitur libellus, Teubner, Leipzig, 1906.

47. Voir N. W. De Witt, 1936 ( «Epicurean contubernium ») p. 57 ; B. Farrington, 1967, p. 12 ; W. Schmid, 1984, p. 72 sq. Les dons pouvaient

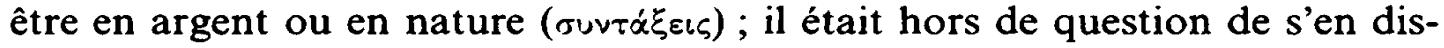

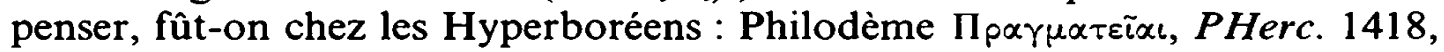
col. XXX, Militello (L. Spina, Il trattato di Filodemo su Epicuro e altri (PHerc. 1418), Cronache Ercolanesi 7, 1977, p. 43-83, ici p. 60) = 184 Us., [121] Arr. ; fr. 182-183 Us. = [123] et [99] Arr. 
parce qu'il y a totalement accédé lui-même. Cette métamorphose du dieu en homme et de l'homme en dieu est possible en Grèce, où la représentation du divin est anthropomorphe et où on honore des hommes-dieux. Elle est possible selon les théories d'Épicure plus que partout ailleurs, puisque Épicure lui-même, fait remarquer De Witt, n'a jamais parlé de l'immortalité des dieux mais seulement de leur incorruptibilité soutenue par la vertu et par l'amitié, une incorruptibilité accessible à l'homme. Elle est possible dans la mesure où l'on admet que l'épicurisme est bien une réforme religieuse ${ }^{48}$, engageant simultanément une vision du monde, une vie individuelle et une communauté humaine nouvelles.

Pour un chrétien comme saint Augustin, l'initiation conférée par le baptême fait accéder la "terre" dont est façonné son être de chair à la forme, à l'effigies, par l'empreinte du sceau divin ${ }^{49}$. L'intuition correcte des dieux, débarrassée de toute superstition, a la même efficacité chez Épicure, elle le transfigure, mais, mieux encore, par l'étroite connexion de la connaissance des dieux et de l'amitié, elle opère, nous allons le voir, une transsubstantiation.

\section{Une autarcie extatique}

Car cette métamorphose demande évidemment à être démontrée dans sa réalisation même. C'est ici qu'il nous faut renvoyer à la proscynèse de Colotès, pour noter la contradiction entre la doctrine de l'autarcie du sage - mais, aussi bien, celle du divin - et l'extase mutuelle de Colotès et d'Épicure,

48. Reconsidération de la religion traditionnelle, non pour la rejeter mais pour lui réinjecter un sens compatible avec une exigence philosophique, après que le platonisme a introduit la religion astrale ? Cette thèse de B. Farrington, 1967, p. 83 sq., permet de rendre compte du rejet quasi obsessionnel des craintes sur l'outre-tombe chez les Épicuriens, et du thème des châtiments et des récompenses, si éloigné des préoccupations de la religion traditionnelle (cf. P. A. Brunt, Philosophy and Religion in the Late Republic, in M. Griffin et J. Barnes (eds), Philosophia togata. Essays on Philosophy and Roman Society, Oxford, 1989, p. 174-198, ici p. 180).

49. Augustin, Confessions I, 18. 
qui se précipitent aux genoux l'un de l'autre et se reconnaissent mutuellement comme dieux; contradiction, plus largement, entre l'accès à la vie paisible des dieux, à cette totale absence de trouble qu'est l'ataraxie, et certain climat d'effusion continuelle que les détracteurs dénoncent chez Épicure et dans sa secte. Si nous réunissons les termes de cette contradiction, nous avons à comprendre comment l'autarcie peut être extatique, ce qui revient à poser la question : comment le plaisir peut-il être philanthropique ? Comment la recherche du congruent à soi peut-elle déboucher sur le dévouement à l'autre?

Nous ne pouvons reprendre ici tout le détail du cheminement à parcourir. La théorie d'Épicure est complexe et le stade où parvient Colotès constitue quasiment le dernier terme de l'initiation à la doctrine. Rappelons d'abord qu'à ce stade ultime toutes les étapes intermédiaires doivent être présentes à l'esprit du myste, grâce à un travail assidu de la mémoire et au caractère entièrement déductif de la doctrine : quand une fois on a saisi l'ensemble des conséquences des propositions premières, et que de cette façon on a bouclé la boucle des connaissances, l'esprit doit être capable de les parcourir tout entières à tout moment, sans plus jamais se laisser troubler par la moindre opinion capable d'ébranler les certitudes acquises; et il se produit comme une brusque entrée en apesanteur : moenia mundi / discedunt, totum uideo per inane geri res (Lucrèce III, 16-17). Voilà que l'âme humaine - cette âme purement matérielle - comme si elle était capable de quitter le corps et de s'élever parmi les dieux, à la manière de Pythagore, d'Archytas ou d'Épiménide - s'installe parmi les dieux, dans la quiétude des intermondes, et peut, comme du bord le rescapé d'un navire en détresse, contempler en pleine sécurité les tourbillons des atomes s'entrechoquant pour former les mondes au hasard dans le vide immense. Épicure parle alors d'une periodeia ${ }^{50}$, une révolution, un tour d'horizon, qui, dans ce contexte d'élévation et d'arrachement aux contingences terrestres, rappelle évidem-

50. Diogène Laërce $X, 36$. 
ment $^{51}$ la purification et l'ascension des âmes, puis leur parcours, dans le cortège d'un dieu, d'une révolution complète du ciel des Idées, où elles se repaissent d'une contemplation béatifique selon le Phèdre de Platon. Mais Épicure dispose, lui, d'autre chose que de l'image d'un attelage pour expliquer cette entrée en apesanteur. Apparet diuom numen sedesque quietae... (Lucrèce, III, 18).

Nous nous sommes posé le problème de la représentation du corps du sage, puisque ce corps est censé pouvoir devenir celui d'un dieu ${ }^{52}$. Résumons: tout le système physique d'Épicure repose sur un certain nombre de lois qui limitent les

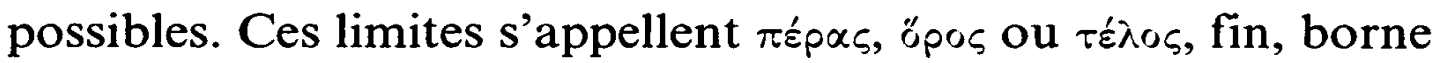
ou perfection. Terminus alte haerens, " une borne profondément enfoncée ", dit encore Lucrèce. Ainsi le nombre des atomes est infini, mais les atomes ne sont pas divisibles, leur taille varie mais non à l'infini, leur forme varie mais non à l'infini. Soit donc le corps d'Épicure : il peut connaître la douleur, mais celle-ci ne saurait dépasser une limite, qui est la mort. Or partout où il n'y a pas douleur, il y a plaisir, le plaisir consistant simplement dans l'absence de douleur. Il y a donc une limite aussi au plaisir, qui est dans la disparition totale de la douleur.

Mais n'existe-t-il pas un plaisir et un bonheur plus grands : celui des dieux ? Pas du tout ! Le plaisir des dieux ne saurait se définir autrement que celui des hommes. L'éternité n'y ajoute rien, au point qu'Épicure n'introduit pas même l'attribut de l'immortalité dans la définition du dieu au début de la Lettre à Ménécée ( " un vivant incorruptible et bienheureux »). "Le temps infini contient un plaisir égal à celui du temps limité, si de ce plaisir on mesure les limites par la raison ", dit la maxime $19^{53}$.

51. N. W. De Witt, 1954 , p. 108 sq. : "The heavenly apocalypse ".

52. On s'est aidée notamment de Jackie Pigeaud sur La maladie de l'âme. Étude sur la relation de l'âme et du corps dans la tradition médicophilosophique antique, Paris, 1989 [1981].

53. Et la maxime 20 ajoute : «La pensée, qui s'est rendu compte de la fin et de la limite de la chair, et qui a fait disparaître les craintes au sujet de l'éternité, procure la vie parfaite, et n'a en rien besoin, en plus, d'un temps infini. " On utilisera désormais pour Épicure les traductions de Marcel Conche, Épicure. Lettres et Maximes, Paris, 1987. 
C'est ainsi que la connaissance du divin parachève la totalité du plaisir du sage en le persuadant que la nature n'a rien à offrir au-delà de ce qu'il possède déjà. Ou plutôt elle en est la condition même, puisque je ne puis atteindre le plaisir total que par la conscience de cette limite en tant que les dieux y sont eux-mêmes assujettis, en tant qu'il s'agit d'une de ces «bornes profondément enfoncées » qui définissent les assises inébranlables de la nature.

Or un dieu, même si je me découvre égal à lui, est d'abord un autre que moi-même. En d'autres termes, la clôture de mon plaisir qui me définissait dans mon unité ne va pouvoir fonctionner comme telle que si elle est en même temps poreuse, perméable à la reconnaissance de l'autre qu'est le dieu.

Du reste que serait un plaisir d'emmuré, un plaisir d'enfermement? Le plaisir catastématique comprend des variations, $\pi \sigma_{c} x_{i}^{\prime} \lambda \lambda \varepsilon \tau x_{l}$, dit la doctrine ${ }^{54}$. Il exige d'être toujours au bord de la dissolution dans le plaisir de l'autre, qui est dieu; il ne peut être atteint que dans la contemplation désirante qui seule maintient la tension sur la limite virtuelle du moi. Il implique donc simultanément ce saut hors de soi, cet élan, cet égarement qui était celui de Colotès. Et l'on peut comprendre alors, en même temps que tous les épanchements d'affect caractéristiques de la secte (voir notre article dans Lalies, 1998), ce raccourci désobligeant de Plutarque (Moralia, $1091 c)$ :

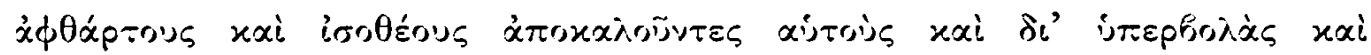

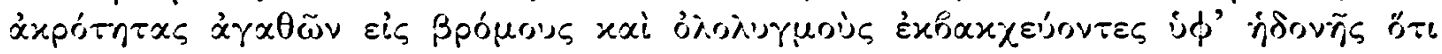

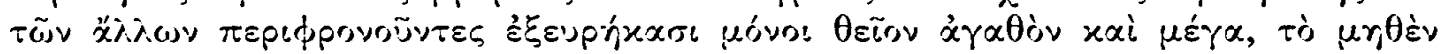
हैy d'égaux des dieux, et, dans l'excès et au plus haut sommet des biens qu'ils reçoivent éclatant de plaisir en un délire bachique de grondements et de youyou parce que, au mépris de tous les autres, ils ont seuls découvert ce grand bien, ce bien divin, à savoir le fait de ne souffrir aucun mal...

54. $K D 18$ : « Le plaisir dans la chair ne peut s'accroître une fois supprimée la douleur du besoin, mais il est seulement varié. La limite du plaisir naît du fait de se rendre compte de ces choses mêmes, et de celles du même genre, qui sont cause pour la pensée des craintes les plus grandes » (trad. Marcel Conche). 
Mais, inversement, la divinité elle-même, en tant qu'elle communique son image, ne se perçoit pas autrement que dans la communication, l'échange ou l'effusion. Il en existe un témoignage naïf : F. Poulsen analysait voici une cinquantaine d'années un buste d'Épicure trafiqué, grâce aux perforations appropriées, de manière à pouvoir offrir l'illusion d'une image parlante $^{55}$. Comme il ne pouvait imaginer que des Épicuriens aient pu se livrer à de pareilles superstitions, l'auteur supposait que ce buste avait dû être réemployé dans un autre culte. Mais nous voyons que cette hypothèse n'est pas du tout nécessaire. L'image d'Épicure divinisé était simplement plus entière et plus convaincante, elle transmettait des «simulacres » plus conformes à la vraie divinité du maître ${ }^{56}$, si on lui faisait transmettre directement ses oracles.

On pourrait objecter qu'Épicure était un dieu très particulier, et que dans ses effusions il devait être bien éloigné de la quiète ataraxie des divinités des intermondes. Rappelons au contraire qu'Épicure ne doit les traits qui le représentent qu'à sa propre ressemblance avec Asklépios ${ }^{57}$, et que cette ressemblance pourrait bien, qu'elle doit même nécessairement impliquer un commerce amical privilégié, à ce niveau-là aussi. Il conviendrait à ce sujet de reprendre l'examen d'un passage de

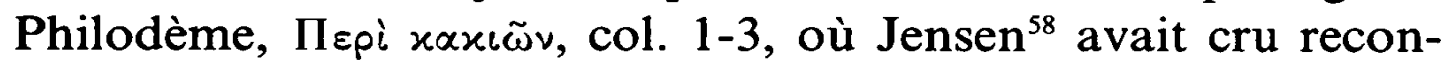
naître une conversation entre Épicure et Asklépios. Sans revoir l'ensemble de la reconstitution de Jensen, qui ne paraît pas défendable ${ }^{59}$, B. Frischer en a contrôlé sur le texte

55. F. Poulsen, Talking, weeping and bleeding sculptures. A chapter of the history of religious fraud, Acta Archaeologica XVI, 1945, p. 178-195.

56. Les statues des dieux devraient initier à l'intellection des divinités véritables : cf. Diogène d'Enanda, fr. 19, Smith, et une phrase de Varron citée par saint Augustin, Cité de Dieu VII, 5, et analysée par P. Boyancé, dans Sur la théologie de Varron, Revue des Études antiques LVII, 1955, p. 57 sq.

57. Voir Jackie Pigeaud, Quel dieu est Épicure ?, Revue des Études latines 50, 1972, p. 139-162 ; W. Fauth, 1973 (cité n. 32).

58. C. Jensen, Ein neuer Brief Epikurs, Abhandlungen $d$. Ges. der Wissenschaft zu Göttingen, philologische-historische Klasse III, 5, Berlin, 1933.

59. Recensions dans R. Philippson, Phil. Woch. 54, 1934, p. 154-160; C. Bailey, $C R 48,1934$, p. 87 ; C. Galavotti, $B F C$ 12, 1934, p. 176-179; voir 
l'hypothèse essentielle: il est selon lui hautement plausible, sinon absolument certain, qu'il s'agisse bien d'un dialogue d'Épicure et du dieu ${ }^{60}$. Ce dialogue avait pu prendre place au cours d'un songe incubatoire, où Asklépios serait apparu en rêve à Épicure, selon les représentations et l'usage diffusés à partir d'Épidaure (nous noterons cependant que la doctrine d'Épicure n'exclut nullement, contrairement à une opinion répandue, des épiphanies divines à des hommes éveillés) ${ }^{61}$. L'intimité qui y apparaît alors entre le sage et le dieu permet de conjecturer, dans leurs échanges, la même extase réciproque que dans le commerce mutuel des amis du Jardin.

Ainsi l'autarcie du plaisir se définit par une extase.

Tâchons maintenant de comprendre plus précisément de quoi est faite cette extase.

Elle est d'abord de contemplation avide. Les attestations ne manquent pas. Rappelons simplement un célèbre passage du De Natura Deorum où Cicéron décrit ainsi la contemplation des dieux selon Épicure:

"Cum maximis uoluptatibus in eas imagines mentem intentam infixamque nostram intelligentiam capere, quae sit et beata natura et aeterna " $(I, 49)$.

C'est avec la plus grande jouissance que notre esprit tendu vers ces simulacres et notre intelligence fixée sur eux saisissent ce qu'est la nature éternelle et bienheureuse.

également E. Bignone, Studi critici sul Testo di Epicuro, SIFC 10, 19321933, p. 93-99 ; J. M. Rist, Epicurus. An Introduction, Cambridge, 1972, p. 160 , n. 1 ; W. Schmid, 1962, col. 707.

60. B. Frischer, 1982, p. 236 sq.

61. Cf. Lucrèce V, 1169 sq. : «Quippe etenim jam tum divom mortalia saecla / egregias animo facies uigante uidebant, / et magis in somnis mirando corporis auctu"; Cicéron, De natura deorum I, 46 : "quae enim forma occurrat umquam aut uigilanti aut dormienti ? "; Philodème, On Piety I, 225-

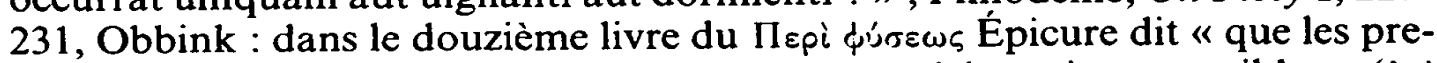
miers hommes sont arrivés au concept d'êtres extérieurs incorruptibles » (̇̇ $\pi \grave{i}$

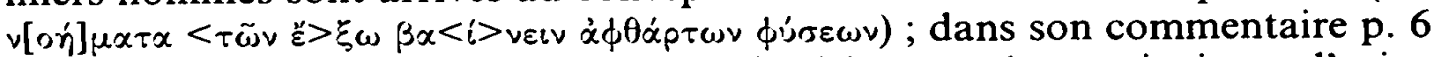
et 306 sq. D. Obbink déclare qu'il s'agit de visions en rêve, mais rien ne l'exige dans le texte, et surtout pas le renvoi qu'il fait à 290 où on lit u $\pi \alpha p$ (qui doit signifier " dans la veille »). Opposer cependant Sextus, $A d v$. dogmat. III (math. IX) 25 et $A d v$. math. IX, 45, où il n'est question que de rêves. Voir Gabriel Droz-Vincent, La théorie épicurienne du rêve, in Scepticisme ex exégèse. Hommage à Camille Pernot, ENS de Fontenay/Saint-Cloud, 1993, p. 165-184. 
Malgré la tension dont parle Cicéron, ce texte évoque une jouissance purement intellectuelle qui, ailleurs, est remplacée par des transports frénétiques; ainsi dans deux passages du traité de Philodème sur les dieux, que nous comprenons ainsi, d'après l'édition de Diels :

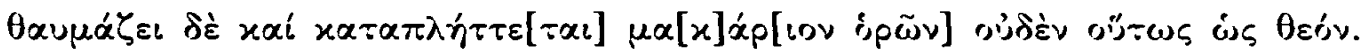

$<$ Le sage > admire, il est bouleversé, quand il ne voit rien d'aussi bienheureux que le dieu (III col. $e$, fr. 86a, 6-8).

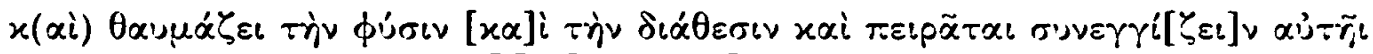

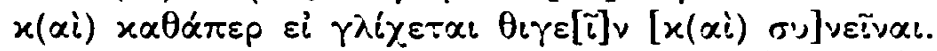

$<$ Le sage > admire la nature et l'être des dieux et cherche à s'en rapprocher, et en quelque sorte il brûle de le toucher et de vivre en sa compagnie (III col. $c$, col. 1, 14-17).

Ces deux extraits commentent à merveille le transport de Colotès, si l'on tient Épicure lui-même pour un dieu.

Et, de fait, l'autarcie extatique se définit également par un désir de ressembler à la divinité et de se confondre avec elle. Cette opération est tenue pour possible au sage; elle a été effectivement réalisée par Épicure ainsi que par tous ses adeptes les plus avancés. La démonstration, dont nous n'indiquons ici que les lignes principales, passe par la théorie de la perception des dieux selon Épicure: percevoir mentalement les dieux dont les simulacres atteignent directement notre âme, grâce à leur finesse, sans passer par le canal de la vue, c'est recevoir en soi les images des dieux et y conformer ses dispositions intérieures $(\delta: \dot{x} \theta \varepsilon \sigma \iota \varsigma)$, ce qui ne peut se faire d'ailleurs qu'une fois que notre âme a été, au préalable, correctement disposée à la réception par une formation pertinente, c'est-à-dire par la physiologie d'Épicure.

Mais cette théorie de la perception des dieux passe ellemême par une théorie de la physique des dieux. Résumons en quelques phrases cette question épineuse ${ }^{62}$, dont il nous

62. On en trouve une très utile récapitulation (pour les années antérieures) dans Jean Bollack, La pensée du plaisir, Paris, 1975, p. 217-238; plus récemment, Michel Malherbe, La théologie matérialiste d'Epicure, Archives de philosophie 40, 1977, p. 363-377; José Kany-Turpin, Cicéron lecteur d'Épicure, Revue philosophique de la France et de l'étranger I, 1986, p. 39-58 ; 
semble que les divers éléments s'expliqueraient sans trop de peine à partir de notre réflexion sur l'amitié fondatrice du Jardin. Tout tourne autour de deux maîtres-mots : oixeıoñ $\sigma \alpha_{\imath}$ et ¿uocoĩ $\sigma \theta x$, " rendre propre à soi " et "être rendu semblable à ", à quoi il faudrait ajouter l'expression constante d'une réciprocité. En effet, les dieux sont faits (puisqu'ils nous sont perceptibles directement par l'âme) d'une texture fine et rare, tissée d'atomes extrêmement mobiles. Cela implique une extrême émotivité apparemment peu compatible avec l'ataraxie. Mais si l'on y ajoute une extrême vigilance et l'excellence dans l'exercice des vertus, on arrive à ceci, que les dieux n'admettent jamais aucune idée fausse qui puisse compromettre leur ataraxie. Donc, ils atteignent à la plénitude du bonheur, mais une plénitude non moins poreuse que celle que nous avions définie plus haut pour le sage : poreuse à quoi ? à ce qui leur est propre et convenable (íócv, oixeiov), autrement dit à leurs semblables (óuoco々). Mais pour que cette porosité à l'autre ajoute au bonheur, il faut qu'elle se nomme amitié, et qu'elle soit mutuelle. Les dieux forment entre eux, par petites sociétés d'amis, des confréries épicuriennes... C'est ainsi qu'il faut comprendre une phrase énigmatique du début de la Lettre à Ménécée $(\S 124)$ :

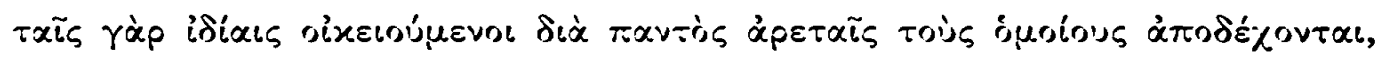

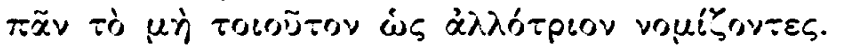

C'est en effet par une continuelle opération d'appropriation au moyen de leurs vertus particulières qu'ils accueillent leurs semblables, considérant comme étranger [à eux-mêmes] tout ce qui n'est pas tel.

On rencontre dans Philodème une formulation voisine :

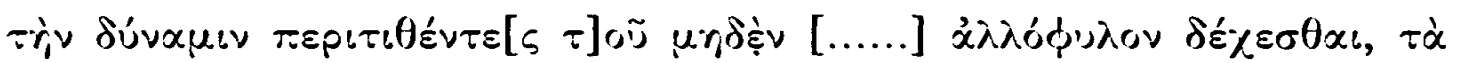

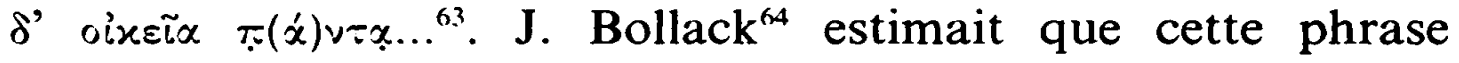
s'inscrit dans un contexte très différent de celui de la lettre à

Jaap Mansfeld, Aspects of Epicurean Theology, Mnemosyne 4 , 46, 1993, p. $172-210$; P. G. Woodward, Star Gods in Philodemus, PHerc. 152/157, Cronache Ercolanesi 19,1989, p. 29-48 ; et D. Obbink, 1996, commentaires aux col. 2-8, 12, 16. La discussion ne paraît pas close.

63. Philodème, Des dieux III, fr. 18, Diels : "Attribuant la faculté de ne rien accueillir d'étranger, mais tout ce qui est propre... »

64. Jean Bollack, 1975, p. 103. 
Ménécée : la question de l'alimentation physique des dieux. Il commentait : « Pour que le rapprochement, même légitime, ait une signification quelconque, il faudrait établir une analogie rigoureuse entre la relation des dieux et des hommes et l'alimentation des figures divines. " Nous cherchons justement à établir une telle analogie. Retenons encore à cet effet cette phrase de Philodème qui fait reposer la nourriture des dieux, et simultanément leur bonheur, sur un commerce mutuel :

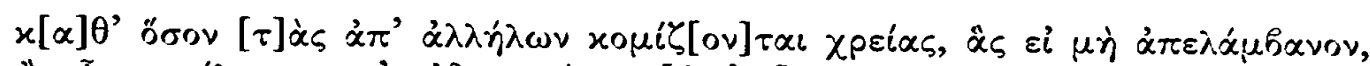

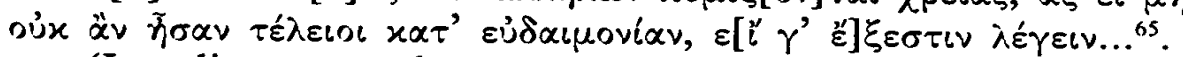

(Les dieux sans doute se soutiennent entre eux) dans la mesure où ils se procurent mutuellement de quoi satisfaire à leurs besoins: s'ils ne recevaient pas cette nourriture leur bonheur resterait inachevé, s'il est permis de s'exprimer ainsi...

Or, les semblables des dieux sont en nombre infini, selon la loi qui veut que tout ce qui existe, existe une infinité de fois ${ }^{66}$. Ainsi, même en circuit fermé, la matière du corps des dieux ne fait jamais défaut ${ }^{67}$, constamment renouvelée par l'extase réciproque de l'amitié, dont la chaîne peut être infinie. Mais d'autre part, chaque dieu est en quelque sorte cloné en une infinité de semblables. Michel Malherbe avait bien résumé cette physique très particulière, sans toutefois la rapporter à l'amitié et au bonheur divin: «La ressemblance seule est capable de compenser la fragilité que le phénomène tient de la matière : celui-ci peut se perdre, s'il se conserve dans son semblable. L'opération de la similitude est cependant complexe, car elle ne l'emporte sur la succession qu'en produisant l'identité... Épicure fait de la ressemblance un principe phy-

65. Philodème, Des dieux III, col. $c$, fr. 84, 1-5, Diels.

66. Loi d'isonomie : loi de proportion et d'équilibre, qui veut qu'à une possibilité donnée d'organisation de la matière, réponde avec une fréquence équivalente (c'est-à-dire nécessairement infinie) la possibilité contraire. Cf. Cicéron, De natura deorum I, 39, 109 et Lucrèce II, 532 sq. : dans l'infini, les forces de destruction sont équilibrées par les forces génératrices. Voir à ce sujet $G$. Freymuth, Eine Anwendung von Epikurs Isonomiegesetz, Philologus 98, 1954, p. 101-115.

67. Selon le processus d'antanaplèrôsis, ou suppeditatio en latin (Lucrèce II, 568). 
sique, puisqu'elle est la source de l'être des dieux. ${ }^{68}$ Le dieu se transforme, ameibei, écrivait Philodème ${ }^{69}$, sans jamais cesser de se rattacher à son origine par le mécanisme de la similitude. Son autarcie extatique ne cesse de s'aliéner dans le semblable, et à chaque instant se parachève dans le même.

Revenons alors à notre passage de la Lettre à Ménécée. Les semblables dont il est question peuvent être aussi bien les sages, qui brûlent, nous l'avons vu, de se confondre avec les dieux. C'est ainsi que le comprend Marcel Conche ${ }^{70}$ : «Imitant les dieux, nous tendons à leur devenir semblables, et leur devenir semblables, c'est en somme être accueillis parmi eux. Les sages forment société avec les dieux. La crainte fait place à une relation nouvelle où l'on peut dire "les sages amis des dieux et les dieux amis des sages". " ${ }^{71}$

Qu'un seul sage alors ait atteint cette fusion mystique, et il devient capable, en en disposant convenablement un seul autre parmi les hommes, par l'amitié, d'implanter dans l'humanité le principe de l'économie divine. "L'amitié mène sa ronde autour du monde, comme un héraut nous appelant à nous réveiller pour nous estimer bienheureux", dit la Sentence 52. Les dieux font entrer le Jardin dans leur propre autarcie, grâce au mystère de l'amitié qui lia, peut-être, Épicure à Asklépios, mais aussi à Métrodore et au petit nombre de ses amis qui accédèrent à sa divinité. C'est ainsi que le transport mutuel d'Épicure et de Colotès peut nous apparaître comme le paradigme d'une transsubstantiation.

Dionysius episcopus cité par Eusèbe, Préparation évangélique XIV, 27, 8, p. $782 c(=$ fr. 364 Us.) résume ainsi le cheminement d'Épicure :

Soit pour s'être penché par-dessus l'extrême limite de l'univers et pour avoir franchi l'enceinte du ciel, soit pour en être sorti par des por-

68. Michel Malherbe, 1977, p. 376.

69. Des dieux III, col. 10, 36-38 et 11, 1-2, Diels : "Néanmoins, un tel dieu se transforme selon la manière qui a été dite puisque, formé d'éléments mêmes, il les échange sans cesse pour d'autres, se rattachant ainsi à ses principes durant toute la succession des temps. "

70. Marcel Conche, 1987, p. 48.

71. Philodème $=386$ Us. 
tes secrètes connues de lui seul, Épicure a pu voir les dieux dans le vide, il a célébré leur vie de délices, puis, pris du désir de leur plaisir et jaloux de cette existence dans le vide, c'est comme cela qu'il a invité tous les gens à participer à cette béatitude en se rendant semblables à ces dieux-là...

«En se rendant semblables»: nous pouvons comprendre alors que les portraits d'Épicure, de Métrodore ou d'Hermarque soient souvent indiscernables. Et saisir le rôle fétiche de leurs images multipliées pour rappeler partout leurs adeptes à la mémoire de leur perfection. "Je ne pourrais pas oublier Épicure, dit Atticus, même si je le voulais : son image m'atteint partout... ${ }^{72}$

Les dieux, ce qu'il y a de plus fluide, de plus changeant, de plus instable, simples images d'eux-mêmes, deviennent alors le support et l'agent de la seule chose qu'Épicure ait appelée immortelle : à savoir l'amitié ${ }^{73}$.

82, av. Édouard-Depreux

F - 92290 Châtenay-Malabry

72. De finibus $\mathrm{V}, 1,3$.

73. "L'homme bien né s'adonne surtout à la sagesse et à l'amitié : desquelles l'une est un bien mortel, l'autre un bien immortel", dit la Sentence 78, qui donne lieu à d'infinies discussions sur la question de savoir si c'est l'amitié ou la sagesse qui doit être dite immortelle : mais nous venons de le voir, l'extase de l'amitié est la condition de l'immortalité des dieux. 